\title{
Learning word-like units from joint audio-visual analysis
}

\author{
David Harwath and James Glass \\ Computer Science and Artificial Intelligence Laboratory \\ Massachusetts Institute of Technology \\ Cambridge, MA 02139, USA \\ \{dharwath, glass\}@mit.edu
}

\begin{abstract}
Given a collection of images and spoken audio captions, we present a method for discovering word-like acoustic units in the continuous speech signal and grounding them to semantically relevant image regions. For example, our model is able to detect spoken instances of the words "lighthouse" within an utterance and associate them with image regions containing lighthouses. We do not use any form of conventional automatic speech recognition, nor do we use any text transcriptions or conventional linguistic annotations. Our model effectively implements a form of spoken language acquisition, in which the computer learns not only to recognize word categories by sound, but also to enrich the words it learns with semantics by grounding them in images.
\end{abstract}

\section{Introduction}

\subsection{Problem Statement and Motivation}

Automatically discovering words and other elements of linguistic structure from continuous speech has been a longstanding goal in computational linguists, cognitive science, and other speech processing fields. Practically all humans acquire language at a very early age, but this task has proven to be an incredibly difficult problem for computers. While conventional automatic speech recognition (ASR) systems have a long history and have recently made great strides thanks to the revival of deep neural networks (DNNs), their reliance on highly supervised training paradigms has essentially restricted their application to the major languages of the world, accounting for a small fraction of the more than 7,000 human languages spoken worldwide (Lewis et al., 2016). The main reason for this limitation is the fact that these supervised approaches require enormous amounts of very expensive human transcripts. Moreover, the use of the written word is a convenient but limiting convention, since there are many oral languages which do not even employ a writing system. In constrast, infants learn to communicate verbally before they are capable of reading and writing - so there is no inherent reason why spoken language systems need to be inseparably tied to text.

The key contribution of this paper has two facets. First, we introduce a methodology capable of not only discovering word-like units from continuous speech at the waveform level with no additional text transcriptions or conventional speech recognition apparatus. Instead, we jointly learn the semantics of those units via visual associations. Although we evaluate our algorithm on an English corpus, it could conceivably run on any language without requiring any text or associated ASR capability. Second, from a computational perspective, our method of speech pattern discovery runs in linear time. Previous work has presented algorithms for performing acoustic pattern discovery in continuous speech (Park and Glass, 2008; Jansen et al., 2010; Jansen and Van Durme, 2011) without the use of transcriptions or another modality, but those algorithms are limited in their ability to scale by their inherent $O\left(n^{2}\right)$ complexity, since they do an exhaustive comparison of the data against itself. Our method leverages correlated information from a second modality - the visual domain - to guide the discovery of words and phrases. This enables our method to run in $O(n)$ time, and we demonstrate it scalability by discovering acoustic patterns in over 522 hours of audio.

\subsection{Previous Work}

A sub-field within speech processing that has garnered much attention recently is unsupervised 
speech pattern discovery. Segmental Dynamic Time Warping (S-DTW) was introduced by Park and Glass (2008), which discovers repetitions of the same words and phrases in a collection of untranscribed acoustic data. Many subsequent efforts extended these ideas (Jansen et al., 2010; Jansen and Van Durme, 2011; Dredze et al., 2010; Harwath et al., 2012; Zhang and Glass, 2009). Alternative approaches based on Bayesian nonparametric modeling (Lee and Glass, 2012; Ondel et al., 2016) employed a generative model to cluster acoustic segments into phoneme-like categories, and related works aimed to segment and cluster either reference or learned phonemelike tokens into higher-level units (Johnson, 2008; Goldwater et al., 2009; Lee et al., 2015).

While supervised object detection is a standard problem in the vision community, several recent works have tackled the problem of weaklysupervised or unsupervised object localization (Bergamo et al., 2014; Cho et al., 2015; Zhou et al., 2015; Cinbis et al., 2016). Although the focus of this work is discovering acoustic patterns, in the process we jointly associate the acoustic patterns with clusters of image crops, which we demonstrate capture visual patterns as well.

The computer vision and NLP communities have begun to leverage deep learning to create multimodal models of images and text. Many works have focused on generating annotations or text captions for images (Socher and $\mathrm{Li}, 2010$; Frome et al., 2013; Socher et al., 2014; Karpathy et al., 2014; Karpathy and Li, 2015; Vinyals et al., 2015; Fang et al., 2015; Johnson et al., 2016). One interesting intersection between word induction from phoneme strings and multimodal modeling of images and text is that of Gelderloos and Chrupaa (2016), who uses images to segment words within captions at the phoneme string level. Other work has taken these ideas beyond text, and attempted to relate images to spoken audio captions directly at the waveform level (Roy, 2003; Harwath and Glass, 2015; Harwath et al., 2016). The work of (Harwath et al., 2016) is the most similar to ours, in which the authors learned embeddings at the entire image and entire spoken caption level and then used the embeddings to perform bidirectional retrieval. In this work, we go further by automatically segmenting and clustering the spoken captions into individual word-like units, as well as the images into object-like categories.

\section{Experimental Data}

We employ a corpus of over 200,000 spoken captions for images taken from the Places205 dataset (Zhou et al., 2014), corresponding to over 522 hours of speech data. The captions were collected using Amazon's Mechanical Turk service, in which workers were shown images and asked to describe them verbally in a free-form manner. The data collection scheme is described in detail in Harwath et al. (2016), but the experiments in this paper leverage nearly twice the amount of data. For training our multimodal neural network as well as the pattern discovery experiments, we use a subset of 214,585 image/caption pairs, and we hold out a set of 1,000 pairs for evaluating the multimodal network's retrieval ability. Because we lack ground truth text transcripts for the data, we used Google's Speech Recognition public API to generate proxy transcripts which we use when analyzing our system. Note that the ASR was only used for analysis of the results, and was not involved in any of the learning.

\section{Audio-Visual Embedding Neural Networks}

We first train a deep multimodal embedding network similar in spirit to the one described in Harwath et al. (2016), but with a more sophisticated architecture. The model is trained to map entire image frames and entire spoken captions into a shared embedding space; however, as we will show, the trained network can then be used to localize patterns corresponding to words and phrases within the spectrogram, as well as visual objects within the image by applying it to small sub-regions of the image and spectrogram. The model is comprised of two branches, one which takes as input images, and the other which takes as input spectrograms. The image network is formed by taking the off-the-shelf VGG 16 layer network (Simonyan and Zisserman, 2014) and replacing the softmax classification layer with a linear transform which maps the 4096-dimensional activations of the second fully connected layer into our 1024-dimensional multimodal embedding space. In our experiments, the weights of this projection layer are trained, but the layers taken from the VGG network below it are kept fixed. The second branch of our network analyzes speech spectrograms as if they were black and white images. Our spectrograms are computed using $40 \log \mathrm{Mel}$ 
filterbanks with a $25 \mathrm{~ms}$ Hamming window and a $10 \mathrm{~ms}$ shift. The input to this branch always has 1 color channel and is always 40 pixels high (corresponding to the $40 \mathrm{Mel}$ filterbanks), but the width of the spectrogram varies depending upon the duration of the spoken caption, with each pixel corresponding to approximately 10 milliseconds worth of audio. The architecture we use is entirely convolutional and shown below, where $\mathrm{C}$ denotes the number of convolutional channels, $\mathrm{W}$ is filter width, $\mathrm{H}$ is filter height, and $\mathrm{S}$ is pooling stride.

1. Convolution: $\mathrm{C}=128, \mathrm{~W}=1, \mathrm{H}=40$, ReLU

2. Convolution: $\mathrm{C}=256, \mathrm{~W}=11, \mathrm{H}=1, \mathrm{ReLU}$

3. Maxpool: $\mathrm{W}=3, \mathrm{H}=1, \mathrm{~S}=2$

4. Convolution: $\mathrm{C}=512, \mathrm{~W}=17, \mathrm{H}=1, \mathrm{ReLU}$

5. Maxpool: $\mathrm{W}=3, \mathrm{H}=1, \mathrm{~S}=2$

6. Convolution: $\mathrm{C}=512, \mathrm{~W}=17, \mathrm{H}=1, \mathrm{ReLU}$

7. Maxpool: $\mathrm{W}=3, \mathrm{H}=1, \mathrm{~S}=2$

8. Convolution: $\mathrm{C}=1024, \mathrm{~W}=17, \mathrm{H}=1$, ReLU

9. Meanpool over entire caption

10. L2 normalization

In practice during training, we restrict the caption spectrograms to all be 1024 frames wide (i.e., $10 \mathrm{sec}$ of speech) by applying truncation or zero padding. Additionally, both the images and spectrograms are mean normalized before training. The overall multimodal network is formed by tying together the image and audio branches with a layer which takes both of their output vectors and computes an inner product between them, representing the similarity score between a given image/caption pair. We train the network to assign high scores to matching image/caption pairs, and lower scores to mismatched pairs.

Within a minibatch of $B$ image/caption pairs, let $S_{j}^{p}, j=1, \ldots, B$ denote the similarity score of the $j^{\text {th }}$ image/caption pair as output by the neural network. Next, for each pair we randomly sample one impostor caption and one impostor image from the same minibatch. Let $S_{j}^{i}$ denote the similarity score between the $j^{\text {th }}$ caption and its impostor image, and $S_{j}^{c}$ be the similarity score between the $j^{\text {th }}$ image and its impostor caption. The total loss for the entire minibatch is then computed as

$$
\begin{aligned}
\mathcal{L}(\theta)=\sum_{j=1}^{B}[\max & \left(0, S_{j}^{c}-S_{j}^{p}+1\right) \\
& \left.+\max \left(0, S_{j}^{i}-S_{j}^{p}+1\right)\right]
\end{aligned}
$$

We train the neural network with 50 epochs of stochastic gradient descent using a batch size $B=$
128 , a momentum of 0.9 , and a learning rate of $1 \mathrm{e}-$ 5 which is set to geometrically decay by a factor between 2 and 5 every 5 to 10 epochs.

\section{Finding and Clustering Audio-Visual Caption Groundings}

Although we have trained our multimodal network to compute embeddings at the granularity of entire images and entire caption spectrograms, we can easily apply it in a more localized fashion. In the case of images, we can simply take any arbitrary crop of an original image and resize it to $224 \times 224$ pixels. The audio network is even more trivial to apply locally, because it is entirely convolutional and the final mean pooling layer ensures that the output will be a 1024-dim vector no matter the extent of the input. The bigger question is where to locally apply the networks in order to discover meaningful acoustic and visual patterns.

Given an image and its corresponding spoken audio caption, we use the term grounding to refer to extracting meaningful segments from the caption and associating them with an appropriate subregion of the image. For example, if an image depicted a person eating ice cream and its caption contained the spoken words "A person is enjoying some ice cream," an ideal set of groundings would entail the acoustic segment containing the word "person" linked to a bounding box around the person, and the segment containing the word "ice cream" linked to a box around the ice cream. We use a constrained brute force ranking scheme to evaluate all possible groundings (with a restricted granularity) between an image and its caption. Specifically, we divide the image into a grid, and extract all of the image crops whose boundaries sit on the grid lines. Because we are mainly interested in extracting regions of interest and not high precision object detection boxes, to keep the number of proposal regions under control we impose several restrictions. First, we use a $10 \times 10$ grid on each image regardless of its original size. Second, we define minimum and maximum aspect ratios as 2:3 and 3:2 so as not to introduce too much distortion and also to reduce the number of proposal boxes. Third, we define a minimum bounding width as $30 \%$ of the original image width, and similarly a minimum height as $30 \%$ of the original image height. In practice, this results in a few thousand proposal regions per image.

To extract proposal segments from the audio 
caption spectrogram, we similarly define a 1-dim grid along the time axis, and consider all possible start/end points at 10 frame (pixel) intervals. We impose minimum and maximum segment length constraints at 50 and 100 frames (pixels), implying that our discovered acoustic patterns are restricted to fall between 0.5 and 1 second in duration. The number of proposal segments will vary depending on the caption length, and typically number in the several thousands. Note that when learning groundings we consider the entire audio sequence, and do not incorporate the 10sec duration constraint imposed during training.

Once we have extracted a set of proposed visual bounding boxes and acoustic segments for a given image/caption pair, we use our multimodal network to compute a similarity score between each unique image crop/acoustic segment pair. Each triplet of an image crop, acoustic segment, and similarity score constitutes a proposed grounding. A naive approach would be to simply keep the top $N$ groundings from this list, but in practice we ran into two problems with this strategy. First, many proposed acoustic segments capture mostly silence due to pauses present in natural speech. We solve this issue by using a simple voice activity detector (VAD) which was trained on the TIMIT corpus(Garofolo et al., 1993). If the VAD estimates that $40 \%$ or more of any proposed acoustic segment is silence, we discard that entire grounding. The second problem we ran into is the fact that the top of the sorted grounding list is dominated by highly overlapping acoustic segments. This makes sense, because highly informative content words will show up in many different groundings with slightly perturbed start or end times. To alleviate this issue, when evaluating a grounding from the top of the proposal list we compare the interval intersection over union (IOU) of its acoustic segment against all acoustic segments already accepted for further consideration. If the IOU exceeds a threshold of 0.1 , we discard the new grounding and continue moving down the list. We stop accumulating groundings once the scores fall to below $50 \%$ of the top score in the "keep" list, or when 10 groundings have been added to the "keep" list. Figure 1 displays a pictorial example of our grounding procedure.

Once we have completed the grounding procedure, we are left with a small set of regions of interest in each image and caption spectrogram.
We use the respective branches of our multimodal network to compute embedding vectors for each grounding's image crop and acoustic segment. We then employ $k$-means clustering separately on the collection of image embedding vectors as well as the collection of acoustic embedding vectors. The last step is to establish an affinity score between each image cluster $\mathcal{I}$ and each acoustic cluster $\mathcal{A}$; we do so using the equation

$$
\operatorname{Affinity}(\mathcal{I}, \mathcal{A})=\sum_{\mathbf{i} \in \mathcal{I}} \sum_{\mathbf{a} \in \mathcal{A}} \mathbf{i}^{\top} \mathbf{a} \cdot \operatorname{Pair}(\mathbf{i}, \mathbf{a})
$$

where $\mathbf{i}$ is an image crop embedding vector, $\mathbf{a}$ is an acoustic segment embedding vector, and $\operatorname{Pair}(\mathbf{i}, \mathbf{a})$ is equal to 1 when $\mathbf{i}$ and a belong to the same grounding pair, and 0 otherwise. After clustering, we are left with a set of acoustic pattern clusters, a set of visual pattern clusters, and a set of linkages describing which acoustic clusters are associated with which image clusters. In the next section, we investigate these clusters in more detail.

\section{Experiments and Analysis}

Table 1: Results for image search and annotation on the Places audio caption data (214k training pairs, $1 \mathrm{k}$ testing pairs). Recall is shown for the top 1,5 , and 10 hits. The model we use in this paper is compared against the meanpool variant of the model architecture presented in Harwath et al. (2016). For both training and testing, the captions were truncated/zero-padded to 10 seconds.

\begin{tabular}{llll}
\hline \multirow{2}{*}{ Model } & \multicolumn{3}{c}{ Search } \\
(Harwath et al., 2016) & 0.090 & 0.261 & 0.372 \\
This work (audio) & 0.112 & 0.312 & 0.431 \\
This work (text) & 0.111 & 0.383 & 0.525 \\
\hline & \multicolumn{3}{c}{ Annotation } \\
Model & R@1 & R@5 & R@ 10 \\
\cline { 2 - 4 } (Harwath et al., 2016) & 0.098 & 0.266 & 0.352 \\
This work (audio) & 0.120 & 0.307 & 0.438 \\
This work (text) & 0.113 & 0.341 & 0.493 \\
\hline
\end{tabular}

We trained our multimodal network on a set of 214,585 image/caption pairs, and vetted it with an image search (given caption, find image) and annotation (given image, find caption) task similar to the one used in Harwath et al. (2016); Karpathy et al. (2014); Karpathy and Li (2015). The image annotation and search recall scores on a 1,000 image/caption pair held-out test set are shown in Table 1. Also shown in this table are the scores 

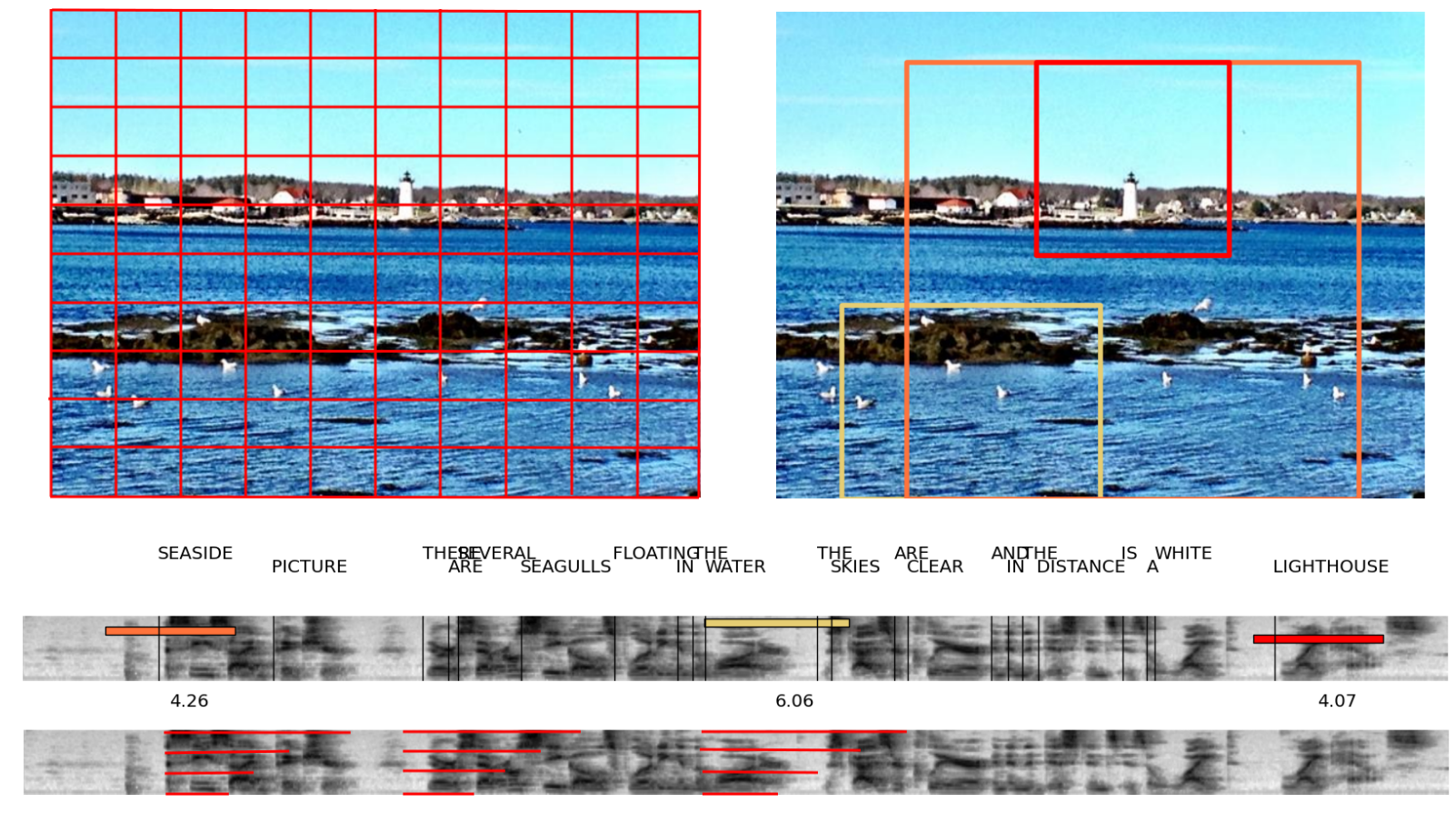

Figure 1: An example of our grounding method. The left image displays a grid defining the allowed start and end coordinates for the bounding box proposals. The bottom spectrogram displays several audio region proposals drawn as the families of stacked red line segments. The image on the right and spectrogram on the top display the final output of the grounding algorithm. The top spectrogram also displays the time-aligned text transcript of the caption, so as to demonstrate which words were captured by the groundings. In this example, the top 3 groundings have been kept, with the colors indicating the audio segment which is grounded to each bounding box.

\begin{tabular}{ccccc}
\hline Word & Count & & Word & Count \\
\cline { 1 - 2 } \cline { 4 - 5 } ocean & 2150 & & castle & 766 \\
(silence) & 127 & & (silence) & 70 \\
the ocean & 72 & & capital & 39 \\
blue ocean & 29 & & large castle & 24 \\
body ocean & 22 & & castles & 23 \\
oceans & 16 & & (noise) & 21 \\
ocean water & 16 & & council & 13 \\
(noise) & 15 & & stone castle & 12 \\
of ocean & 14 & & capitol & 10 \\
oceanside & 14 & old castle & 10 \\
\hline
\end{tabular}

Table 2: Examples of the breakdown of word/phrase identities of several acoustic clusters

achieved by a model which uses the ASR text transcriptions for each caption instead of the speech audio. The text captions were truncated/padded to 20 words, and the audio branch of the network was replaced with a branch with the following architecture:

1. Word embedding layer of dimension 200
2. Temporal Convolution: $\mathrm{C}=512, \mathrm{~W}=3, \mathrm{ReLU}$

3. Temporal Convolution: $\mathrm{C}=1024, \mathrm{~W}=3$

4. Meanpool over entire caption

5. L2 normalization

One would expect that access to ASR hypotheses should improve the recall scores, but the performance gap is not enormous. Access to the ASR hypotheses provides a relative improvement of approximately $21.8 \%$ for image search R@10 and $12.5 \%$ for annotation R@ 10 compared to using no transcriptions or ASR whatsoever.

We performed the grounding and pattern clustering steps on the entire training dataset, which resulted in a total of $1,161,305$ unique grounding pairs. For evaluation, we wish to assign a label to each cluster and cluster member, but this is not completely straightforward since each acoustic segment may capture part of a word, a whole word, multiple words, etc. Our strategy is to forcealign the Google recognition hypothesis text to the audio, and then assign a label string to each acoustic segment based upon which words it overlaps in time. The alignments are created with the help of a Kaldi (Povey et al., 2011) speech recognizer 
Table 3: Top 50 clusters with $k=500$ sorted by increasing variance. Legend: $\left|C_{c}\right|$ is acoustic cluster size, $\left|C_{i}\right|$ is associated image cluster size, Pur. is acoustic cluster purity, $\sigma^{2}$ is acoustic cluster variance, and Cov. is acoustic cluster coverage. A dash (-) indicates a cluster whose majority label is silence.

\begin{tabular}{|c|c|c|c|c|c|c|c|c|c|c|c|}
\hline Trans & $\left|C_{c}\right|$ & $\left|C_{i}\right|$ & Pur. & $\sigma^{2}$ & Cov. & Trans & $\left|C_{c}\right|$ & $\left|C_{i}\right|$ & Pur. & $\sigma^{2}$ & Cov. \\
\hline - & 1059 & 3480 & 0.70 & 0.26 & - & snow & 4331 & 3480 & 0.85 & 0.26 & 0.45 \\
\hline desert & 1936 & 2896 & 0.82 & 0.27 & 0.67 & kitchen & 3200 & 2990 & 0.88 & 0.28 & 0.76 \\
\hline restaurant & 1921 & 2536 & 0.89 & 0.29 & 0.71 & mountain & 4571 & 2768 & 0.86 & 0.30 & 0.38 \\
\hline black & 4369 & 2387 & 0.64 & 0.30 & 0.17 & skyscraper & 843 & 3205 & 0.84 & 0.30 & 0.84 \\
\hline bridge & 1654 & 2025 & 0.84 & 0.30 & 0.25 & tree & 5303 & 3758 & 0.90 & 0.30 & 0.16 \\
\hline castle & 1298 & 2887 & 0.72 & 0.31 & 0.74 & bridge & 2779 & 2025 & 0.81 & 0.32 & 0.41 \\
\hline & 2349 & 2165 & 0.31 & 0.33 & - & ocean & 2913 & 3505 & 0.87 & 0.33 & 0.71 \\
\hline table & 3765 & 2165 & 0.94 & 0.33 & 0.23 & windmill & 1458 & 3752 & 0.71 & 0.33 & 0.76 \\
\hline window & 1890 & 2795 & 0.85 & 0.34 & 0.21 & river & 2643 & 3204 & 0.76 & 0.35 & 0.62 \\
\hline water & 5868 & 3204 & 0.90 & 0.35 & 0.27 & beach & 1897 & 2964 & 0.79 & 0.35 & 0.64 \\
\hline flower & 3906 & 2587 & 0.92 & 0.35 & 0.67 & wall & 3158 & 3636 & 0.84 & 0.35 & 0.23 \\
\hline sky & 4306 & 6055 & 0.76 & 0.36 & 0.34 & street & 2602 & 2385 & 0.86 & 0.36 & 0.49 \\
\hline golf course & 1678 & 3864 & 0.44 & 0.36 & 0.63 & field & 3896 & 3261 & 0.74 & 0.36 & 0.37 \\
\hline tree & 4098 & 3758 & 0.89 & 0.36 & 0.13 & lighthouse & 1254 & 1518 & 0.61 & 0.36 & 0.83 \\
\hline forest & 1752 & 3431 & 0.80 & 0.37 & 0.56 & church & 2503 & 3140 & 0.86 & 0.37 & 0.72 \\
\hline people & 3624 & 2275 & 0.91 & 0.37 & 0.14 & baseball & 2777 & 1929 & 0.66 & 0.37 & 0.86 \\
\hline field & 2603 & 3922 & 0.74 & 0.37 & 0.25 & car & 3442 & 2118 & 0.79 & 0.38 & 0.27 \\
\hline people & 4074 & 2286 & 0.92 & 0.38 & 0.17 & shower & 1271 & 2206 & 0.74 & 0.38 & 0.82 \\
\hline people walking & 918 & 2224 & 0.63 & 0.38 & 0.25 & wooden & 3095 & 2723 & 0.63 & 0.38 & 0.28 \\
\hline mountain & 3464 & 3239 & 0.88 & 0.38 & 0.29 & tree & 3676 & 2393 & 0.89 & 0.39 & 0.11 \\
\hline- & 1976 & 3158 & 0.28 & 0.39 & - & snow & 2521 & 3480 & 0.79 & 0.39 & 0.24 \\
\hline water & 3102 & 2948 & 0.90 & 0.39 & 0.14 & rock & 2897 & 2967 & 0.76 & 0.39 & 0.26 \\
\hline - & 2918 & 3459 & 0.08 & 0.39 & - & night & 3027 & 3185 & 0.44 & 0.39 & 0.59 \\
\hline station & 2063 & 2083 & 0.85 & 0.39 & 0.62 & chair & 2589 & 2288 & 0.89 & 0.39 & 0.22 \\
\hline building & 6791 & 3450 & 0.89 & 0.40 & 0.21 & city & 2951 & 3190 & 0.67 & 0.40 & 0.50 \\
\hline
\end{tabular}

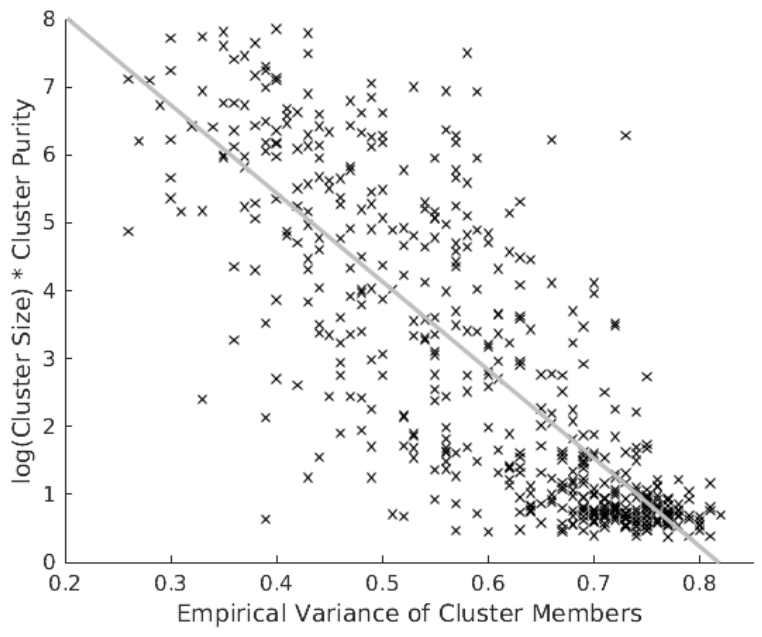

Figure 2: Scatter plot of audio cluster purity weighted by $\log$ cluster size vs variance for $k=$ 500 (least-squares line superimposed).

based on the standard WSJ recipe and trained using the Google ASR hypothesis as a proxy for the transcriptions. Any word whose duration is overlapped $30 \%$ or more by the acoustic segment is included in the label string for the segment. We then employ a majority vote scheme to derive the overall cluster labels. When computing the purity of a cluster, we count a cluster member as matching the cluster label as long as the overall cluster label appears in the member's label string. In other words, an acoustic segment overlapping the words "the lighthouse" would receive credit for matching the overall cluster label "lighthouse". A breakdown of the segments captured by two clusters is shown in Table 2. We investigated some simple schemes for predicting highly pure clusters, and found that the empirical variance of the cluster members (average squared distance to the cluster centroid) was a good indicator. Figure 2 displays a scatter plot of cluster purity weighted by the natural $\log$ of the cluster size against the empirical variance. Large, pure clusters are easily predicted by their low empirical variance, while a high variance is indicative of a garbage cluster.

Ranking a set of $k=500$ acoustic clusters by their variance, Table 3 displays some statistics for the 50 lowest-variance clusters. We see that most of the clusters are very large and highly pure, and their labels reflect interesting object categories being identified by the neural network. We additionally compute the coverage of each cluster by counting the total number of instances of the clus- 


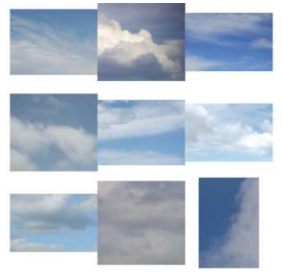

sky

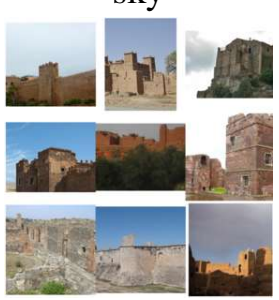

castle

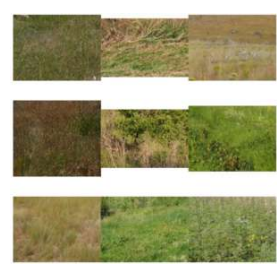

grass

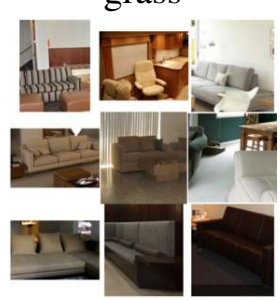

couch

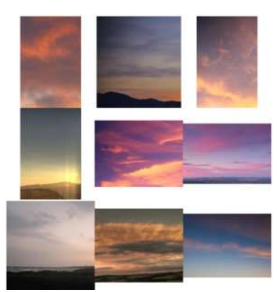

sunset

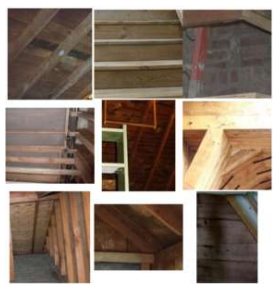

wooden

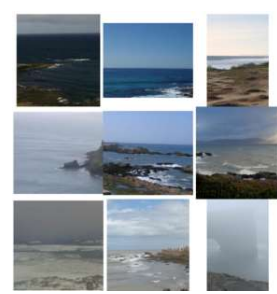

ocean

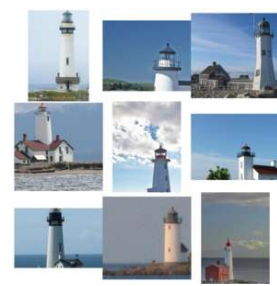

lighthouse

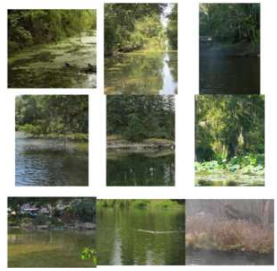

river

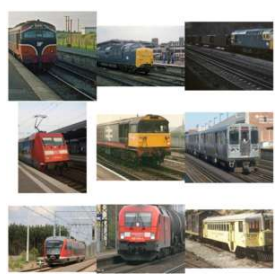

train

Figure 3: The 9 most central image crops from several image clusters, along with the majority-vote label of their most associated acoustic pattern cluster

Table 4: Clustering statistics of the acoustic clusters for various values of $k$ and different settings of the variance-based cluster pruning threshold. Legend: $|\mathcal{C}|=$ number of clusters remaining after pruning, $|\mathcal{X}|$ $=$ number of datapoints after pruning, Pur $=$ purity, $|\mathcal{L}|=$ number of unique cluster labels, $\mathrm{AC}=$ average cluster coverage

\begin{tabular}{|c|c|c|c|c|c|c|c|c|c|c|}
\hline \multirow[b]{2}{*}{$k$} & \multicolumn{5}{|c|}{$\sigma^{2}<0.9$} & \multicolumn{5}{|c|}{$\sigma^{2}<0.65$} \\
\hline & $|\mathcal{C}|$ & $|\mathcal{X}|$ & Pur & $|\mathcal{L}|$ & $\mathrm{AC}$ & $|\mathcal{C}|$ & $|\mathcal{X}|$ & Pur & $|\mathcal{L}|$ & $\mathrm{AC}$ \\
\hline 250 & 249 & 1081514 & .364 & 149 & .423 & 128 & 548866 & .575 & 108 & .463 \\
\hline 500 & 499 & 1097225 & .396 & 242 & .332 & 278 & 623159 & .591 & 196 & .375 \\
\hline 750 & 749 & 1101151 & .409 & 308 & .406 & 434 & 668771 & .585 & 255 & .450 \\
\hline 1000 & 999 & 1103391 & .411 & 373 & .336 & 622 & 710081 & .568 & 318 & .382 \\
\hline 1500 & 1496 & 1104631 & .429 & 464 & .316 & 971 & 750162 & .566 & 413 & .366 \\
\hline 2000 & 1992 & 1106418 & .431 & 540 & .237 & 1354 & 790492 & .546 & 484 & .271 \\
\hline
\end{tabular}

ter label anywhere in the training data, and then compute what fraction of those instances were captured by the cluster. There are many examples of high coverage clusters, e.g. the "skyscraper" cluster captures $84 \%$ of all occurrences of the word "skyscraper", while the "baseball" cluster captures $86 \%$ of all occurrences of the word "baseball". This is quite impressive given the fact that no conventional speech recognition was employed, and neither the multimodal neural network nor the grounding algorithm had access to the text transcripts of the captions.

To get an idea of the impact of the $k$ parameter as well as a variance-based cluster pruning threshold based on Figure 2, we swept $k$ from 250 to 2000 and computed a set of statistics shown in Table 4. We compute the standard overall cluster purity evaluation metric in addition to the average coverage across clusters. The table shows the natural tradeoff between cluster purity and redun- dancy (indicated by the average cluster coverage) as $k$ is increased. In all cases, the variance-based cluster pruning greatly increases both the overall purity and average cluster coverage metrics. We also notice that more unique cluster labels are discovered with a larger $k$.

Next, we examine the image clusters. Figure 3 displays the 9 most central image crops for a set of 10 different image clusters, along with the majority-vote label of each image cluster's associated audio cluster. In all cases, we see that the image crops are highly relevant to their audio cluster label. We include many more example image clusters in Appendix A.

In order to examine the semantic embedding space in more depth, we took the top 150 clusters from the same $k=500$ clustering run described in Table 3 and performed t-SNE (van der Maaten and Hinton, 2008) analysis on the cluster centroid vectors. We projected each centroid down to 2 di- 


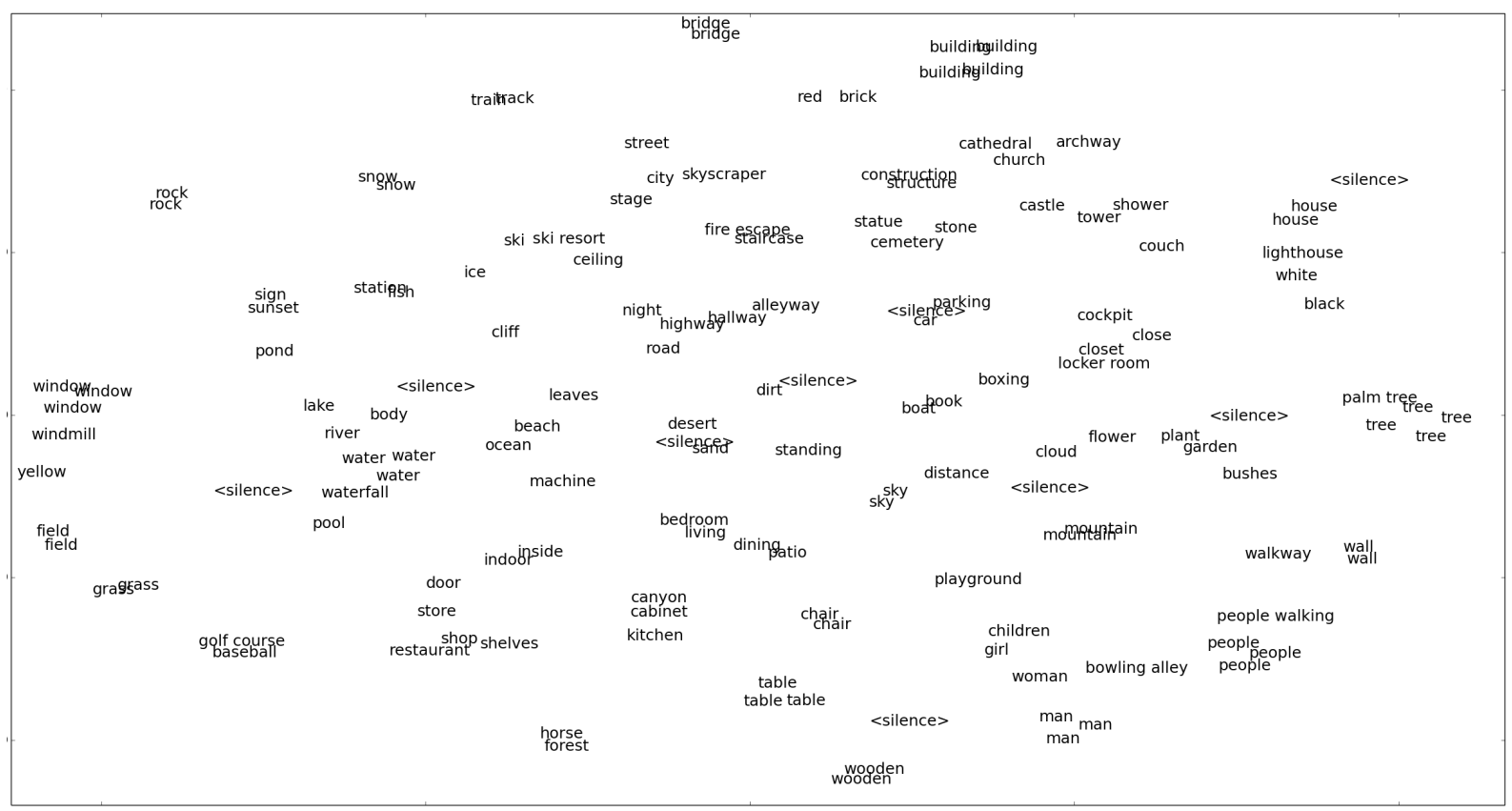

Figure 4: t-SNE analysis of the 150 lowest-variance audio pattern cluster centroids for $k=500$. Displayed is the majority-vote transcription of the each audio cluster. All clusters shown contained a minimum of 583 members and an average of 2482, with an average purity of .668.

mensions and plotted their majority-vote labels in Figure 4. Immediately we see that different clusters which capture the same label closely neighbor one another, indicating that distances in the embedding space do indeed carry information discriminative across word types (and suggesting that a more sophisticated clustering algorithm than $k$ means would perform better). More interestingly, we see that semantic information is also reflected in these distances. The cluster centroids for "lake," "river," "body," "water," "waterfall," "pond," and "pool" all form a tight meta-cluster, as do "restaurant," "store," "shop," and "shelves," as well as "children," "girl," "woman," and "man." Many other semantic meta-clusters can be seen in Figure 4 , suggesting that the embedding space is capturing information that is highly discriminative both acoustically and semantically.

Because our experiments revolve around the discovery of word and object categories, a key question to address is the extent to which the supervision used to train the VGG network constrains or influences the kinds of objects learned. Because the 1,000 object classes from the ILSVRC2012 task (Russakovsky et al., 2015) used to train the VGG network were derived from WordNet synsets (Fellbaum, 1998), we can measure the semantic similarity between the words learned by our network and the ILSVRC2012 class labels by using synset similarity measures within WordNet. We do this by first building a list of the 1,000 WordNet synsets associated with the ILSVRC2012 classes. We then take the set of unique majority-vote labels associated with the discovered word clusters for $k=500$, filtered by setting a threshold on their variance $\left(\sigma^{2} \leq 0.65\right)$ so as to get rid of garbage clusters, leaving us with 197 unique acoustic cluster labels. We then look up each cluster label in WordNet, and compare all noun senses of the label to every ILSVRC2012 class synset according to the path similarity measure. This measure describes the distance between two synsets in a hyponym/hypernym hierarchy, where a score of 1 represents identity and lower scores indicate less similarity. We retain the highest score between any sense of the cluster label and any ILSVRC2012 synset. Of the 197 unique cluster labels, only 16 had a distance of 1 from any ILSVRC12 class, which would indicate an exact match. A path similarity of 0.5 indicates one degree of separation in the hyponym/hypernym hierarchy - for example, the similarity between "desk" and "table" is 0.5 .47 cluster labels were found to have a similarity of 0.5 to some ILSVRC12 class, leaving 134 cluster labels whose highest similarity to any ILSVRC12 class was less than 0.5 . In 
other words, more than two thirds of the highly pure pattern clusters learned by our network were dissimilar to all of the 1,000 ILSVRC12 classes used to pretrain the VGG network, indicating that our model is able to generalize far beyond the set of classes found in the ILSVRC12 data. We display the labels of the 40 lowest variance acoustic clusters labels along with the name and similarity score of their closest ILSVRC12 synset in Table 5.

\begin{tabular}{ccc}
\hline Cluster & ILSVRC synset & Similarity \\
\hline snow & cliff.n.01 & 0.14 \\
desert & cliff.n.01 & 0.12 \\
kitchen & patio.n.01 & 0.25 \\
restaurant & restaurant.n.01 & 1.00 \\
mountain & alp.n.01 & 0.50 \\
black & pool_table.n.01 & 0.25 \\
skyscraper & greenhouse.n.01 & 0.33 \\
bridge & steel_arch_bridge.n.01 & 0.50 \\
tree & daisy.n.01 & 0.14 \\
castle & castle.n.02 & 1.00 \\
ocean & cliff.n.01 & 0.14 \\
table & desk.n.01 & 0.50 \\
windmill & cash_machine.n.01 & 0.20 \\
window & screen.n.03 & 0.33 \\
river & cliff.n.01 & 0.12 \\
water & menu.n.02 & 0.25 \\
beach & cliff.n.01 & 0.33 \\
flower & daisy.n.01 & 0.50 \\
wall & cliff.n.01 & 0.33 \\
sky & cliff.n.01 & 0.11 \\
street & swing.n.02 & 0.14 \\
golf course & swing.n.02 & 0.17 \\
field & cliff.n.01 & 0.20 \\
lighthouse & beacon.n.03 & 1.00 \\
forest & cliff.n.01 & 0.20 \\
church & church.n.02 & 1.00 \\
people & street_sign.n.01 & 0.17 \\
baseball & baseball.n.02 & 1.00 \\
car & freight_car.n.01 & 0.50 \\
shower & swing.n.02 & 0.17 \\
people walking & (none) & 0.00 \\
wooden & (none) & 0.00 \\
rock & toilet_tissue.n.01 & 0.20 \\
night & street_sign.n.01 & 0.14 \\
station & swing.n.02 & 0.20 \\
chair & barber_chair.n.01 & 0.50 \\
building & greenhouse.n.01 & 0.50 \\
city & cliff.n.01 & 0.12 \\
white & jean.n.01 & 0.33 \\
sunset & street_sign.n.01 & 0.11 \\
\hline & &
\end{tabular}

Table 5: The 40 lowest variance, uniquely-labeled acoustic clusters paired with their most similar ILSVRC2012 synset.

\section{Conclusions and Future Work}

In this paper, we have demonstrated that a neural network trained to associate images with the waveforms representing their spoken audio captions can successfully be applied to discover and cluster acoustic patterns representing words or short phrases in untranscribed audio data. An analogous procedure can be applied to visual images to discover visual patterns, and then the two modalities can be linked, allowing the network to learn, for example, that spoken instances of the word "train" are associated with image regions containing trains. This is done without the use of a conventional automatic speech recognition system and zero text transcriptions, and therefore is completely agnostic to the language in which the captions are spoken. Further, this is done in $O(n)$ time with respect to the number of image/caption pairs, whereas previous stateof-the-art acoustic pattern discovery algorithms which leveraged acoustic data alone run in $O\left(n^{2}\right)$ time. We demonstrate the success of our methodology on a large-scale dataset of over 214,000 image/caption pairs comprising over 522 hours of spoken audio data, which is to our knowledge the largest scale acoustic pattern discovery experiment ever performed. We have shown that the shared multimodal embedding space learned by our model is discriminative not only across visual object categories, but also acoustically and semantically across spoken words.

The future directions in which this research could be taken are incredibly fertile. Because our method creates a segmentation as well as an alignment between images and their spoken captions, a generative model could be trained using these alignments. The model could provide a spoken caption for an arbitrary image, or even synthesize an image given a spoken description. Modeling improvements are also possible, aimed at the goal of incorporating both visual and acoustic localization into the neural network itself. The same framework we use here could be extended to video, enabling the learning of actions, verbs, environmental sounds, and the like. Additionally, by collecting a second dataset of captions for our images in a different language, such as Spanish, our model could be extended to learn the acoustic correspondences for a given object category in both languages. This paves the way for creating a speech-to-speech translation model not only with absolutely zero need for any sort of text transcriptions, but also with zero need for directly parallel linguistic data or manual human translations. 


\section{References}

Alessandro Bergamo, Loris Bazzani, Dragomir Anguelov, and Lorenzo Torresani. 2014. Self-taught object localization with deep networks. CoRR abs/1409.3964. http://arxiv.org/abs/1409.3964.

Minsu Cho, Suha Kwak, Cordelia Schmid, and Jean Ponce. 2015. Unsupervised object discovery and localization in the wild: Part-based matching with bottom-up region proposals. In Proceedings of CVPR.

Ramazan Cinbis, Jakob Verbeek, and Cordelia Schmid. 2016. Weakly supervised object localization with multi-fold multiple instance learning. In IEEE Transactions on Pattern Analysis and Machine Intelligence.

Mark Dredze, Aren Jansen, Glen Coppersmith, and Kenneth Church. 2010. NLP on spoken documents without ASR. In Proceedings of EMNLP.

Hao Fang, Saurabh Gupta, Forrest Iandola, Srivastava Rupesh, Li Deng, Piotr Dollar, Jianfeng Gao, Xiaodong He, Margaret Mitchell, Platt John C., C. Lawrence Zitnick, and Geoffrey Zweig. 2015. From captions to visual concepts and back. In Proceedings of CVPR.

Christiane Fellbaum. 1998. WordNet: An Electronic Lexical Database. Bradford Books.

Andrea Frome, Greg S. Corrado, Jonathon Shlens, Samy Bengio, Jeffrey Dean, Marc'Aurelio Ranzato, and Tomas Mikolov. 2013. Devise: A deep visual-semantic embedding model. In Proceedings of the Neural Information Processing Society.

John Garofolo, Lori Lamel, William Fisher, Jonathan Fiscus, David Pallet, Nancy Dahlgren, and Victor Zue. 1993. The TIMIT acoustic-phonetic continuous speech corpus.

Lieke Gelderloos and Grzegorz Chrupaa. 2016. From phonemes to images: levels of representation in a recurrent neural model of visually-grounded language learning. In arXiv:1610.03342.

Sharon Goldwater, Thomas Griffiths, and Mark Johnson. 2009. A Bayesian framework for word segmentation: exploring the effects of context. In Cognition, vol. 112 pp.21-54.

David Harwath and James Glass. 2015. Deep multimodal semantic embeddings for speech and images. In Proceedings of the IEEE Workshop on Automatic Speech Recognition and Understanding.

David Harwath, Timothy J. Hazen, and James Glass. 2012. Zero resource spoken audio corpus analysis. In Proceedings of ICASSP.

David Harwath, Antonio Torralba, and James R. Glass. 2016. Unsupervised learning of spoken language with visual context. In Proceedings of NIPS.

Aren Jansen, Kenneth Church, and Hynek Hermansky. 2010. Toward spoken term discovery at scale with zero resources. In Proceedings of Interspeech.

Aren Jansen and Benjamin Van Durme. 2011. Efficient spoken term discovery using randomized algorithms. In Proceedings of IEEE Workshop on Automatic Speech Recognition and Understanding.
Justin Johnson, Andrej Karpathy, and Li Fei-Fei. 2016. Densecap: Fully convolutional localization networks for dense captioning. In Proceedings of CVPR.

Mark Johnson. 2008. Unsupervised word segmentation for sesotho using adaptor grammars. In Proceedings of $A C L$ SIG on Computational Morphology and Phonology.

Andrej Karpathy, Armand Joulin, and Fei-Fei Li. 2014. Deep fragment embeddings for bidirectional image sentence mapping. In Proceedings of the Neural Information Processing Society.

Andrej Karpathy and Fei-Fei Li. 2015. Deep visual-semantic alignments for generating image descriptions. In Proceedings of CVPR.

Chia-Ying Lee and James Glass. 2012. A nonparametric Bayesian approach to acoustic model discovery. In Proceedings of the 2012 meeting of the Association for Computational Linguistics.

Chia-Ying Lee, Timothy J. O'Donnell, and James Glass. 2015. Unsupervised lexicon discovery from acoustic input. In Transactions of the Association for Computational Linguistics.

M. Paul Lewis, Gary F. Simon, and Charles D. Fennig. 2016. Ethnologue: Languages of the World, Nineteenth edition. SIL International. Online version: http://www.ethnologue.com.

Lucas Ondel, Lukas Burget, and Jan Cernocky. 2016. Variational inference for acoustic unit discovery. In 5th Workshop on Spoken Language Technology for Underresourced Language.

Alex Park and James Glass. 2008. Unsupervised pattern discovery in speech. In IEEE Transactions on Audio, Speech, and Language Processing vol. 16, no.1, pp. 186-197.

Daniel Povey, Arnab Ghoshal, Gilles Boulianne, Lukas Burget, Ondrej Glembek, Nagendra Goel, Mirko Hannemann, Petr Motlicek, Yanmin Qian, Petr Schwarz, Jan Silovsky, Georg Stemmer, and Karel Vesely. 2011. The Kaldi speech recognition toolkit. In IEEE 2011 Workshop on Automatic Speech Recognition and Understanding.

Deb Roy. 2003. Grounded spoken language acquisition: Experiments in word learning. In IEEE Transactions on Multimedia.

Olga Russakovsky, Jia Deng, Hao Su, Jonathan Krause, Sanjeev Satheesh, Sean Ma, Zhiheng Huang, Andrej Karpathy, Aditya Khosla, Michael Bernstein, Alexander C. Berg, and Li Fei-Fei. 2015. ImageNet Large Scale Visual Recognition Challenge. International Journal of Computer Vision (IJCV) 115(3):211-252. https://doi.org/10.1007/s11263-015-0816-y.

Karen Simonyan and Andrew Zisserman. 2014. Very deep convolutional networks for large-scale image recognition. CoRR abs/1409.1556.

Richard Socher, Andrej Karpathy, Quoc V. Le, Christopher D. Manning, and Andrew Y. Ng. 2014. Grounded compositional semantics for finding and describing images with sentences. In Transactions of the Association for Computational Linguistics. 
Richard Socher and Fei-Fei Li. 2010. Connecting modalities: Semi-supervised segmentation and annotation of images using unaligned text corpora. In Proceedings of CVPR.

Laurens van der Maaten and Geoffrey Hinton. 2008. Visualizing high-dimensional data using t-sne. In Journal of Machine Learning Research.

Oriol Vinyals, Alexander Toshev, Samy Bengio, and Dimitru Erhan. 2015. Show and tell: A neural image caption generator. In Proceedings of CVPR.

Yaodong Zhang and James Glass. 2009. Unsupervised spoken keyword spotting via segmental DTW on Gaussian posteriorgrams. In Proceedings ASRU.

Bolei Zhou, Aditya Khosla, Agata Lapedriza, Aude Oliva, and Antonio Torralba. 2015. Object detectors emerge in deep scene CNNs. In Proceedings of ICLR.

Bolei Zhou, Agata Lapedriza, Jianxiong Xiao, Antonio Torralba, and Aude Oliva. 2014. Learning deep features for scene recognition using places database. In Proceedings of the Neural Information Processing Society. 
A Additional Cluster Visualizations

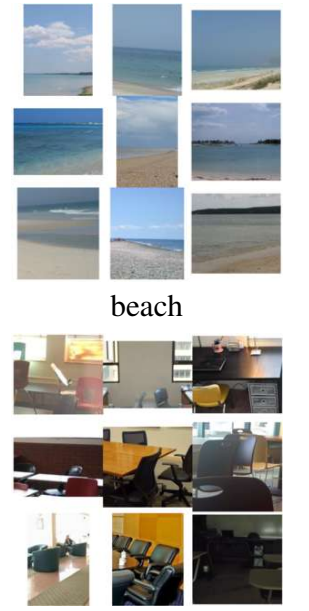

chair

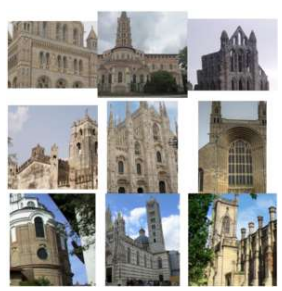

church

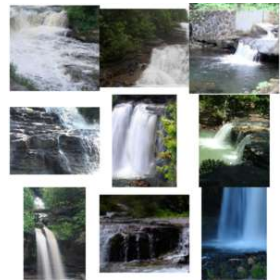

waterfall

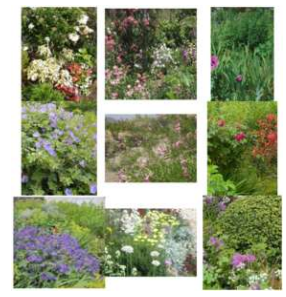

flowers

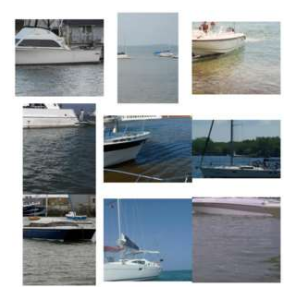

boat

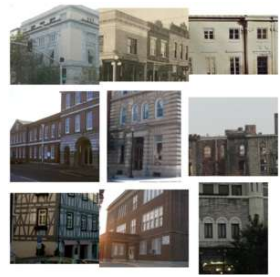

building

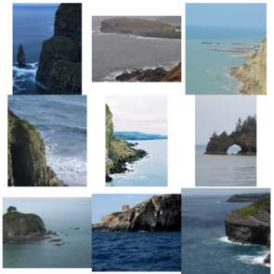

cliff

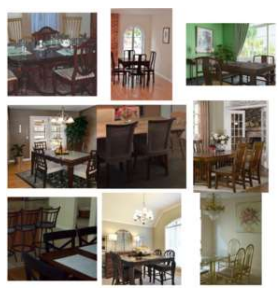

table

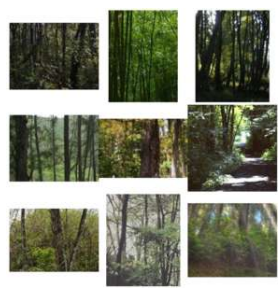

forest

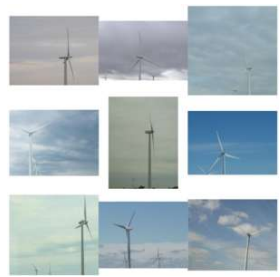

windmills

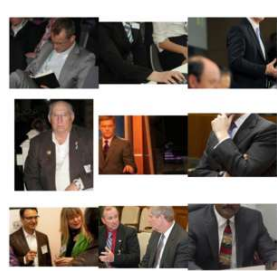

man

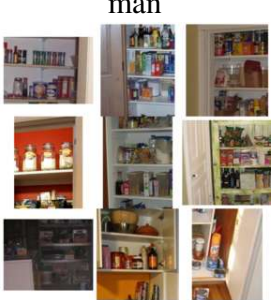

shelves

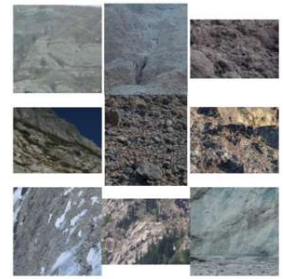

rock

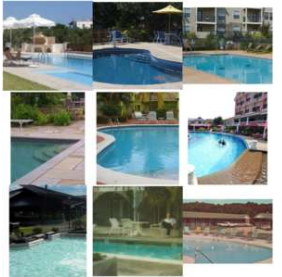

pool

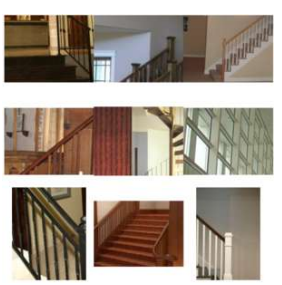

staircase

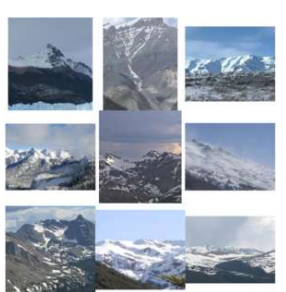

mountain
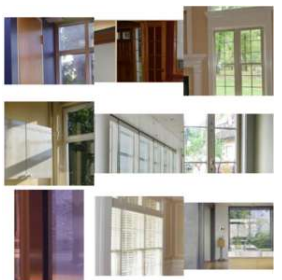

window

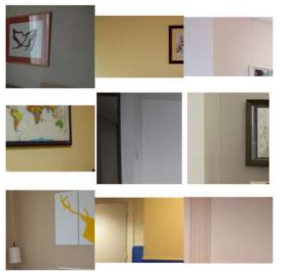

wall

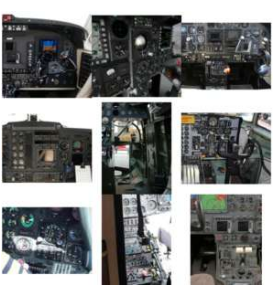

cockpit

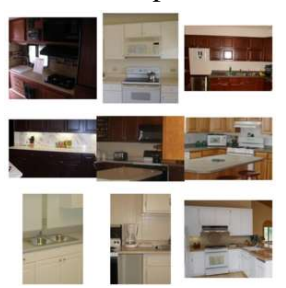

kitchen

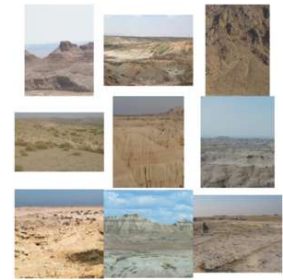

desert

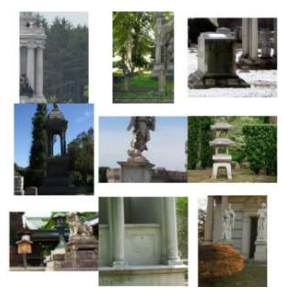

statue

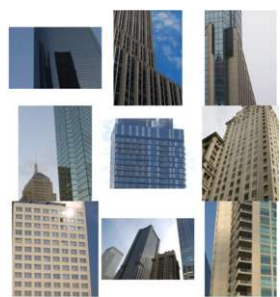

skyscraper

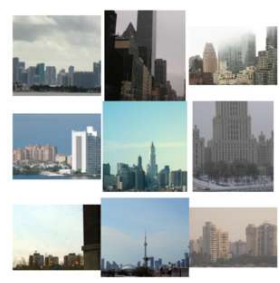

city
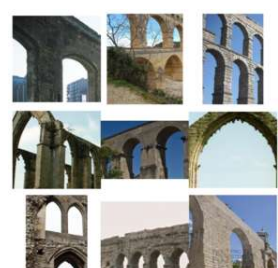

archway

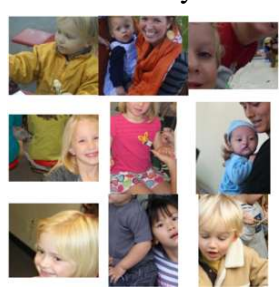

girl

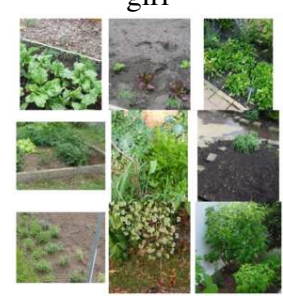

plant

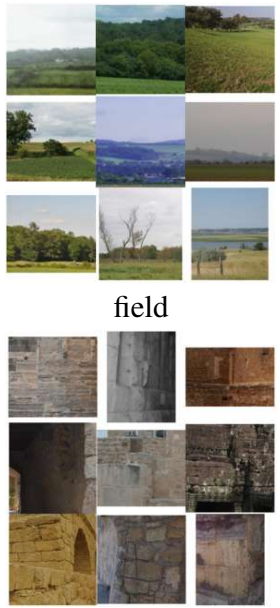

stone

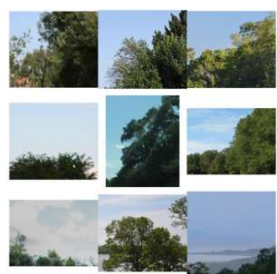

trees

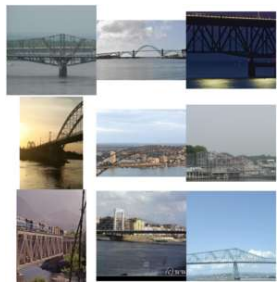
bridge

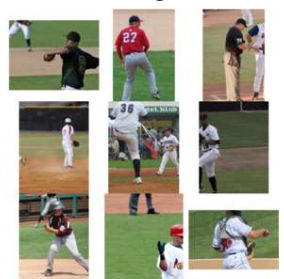

baseball

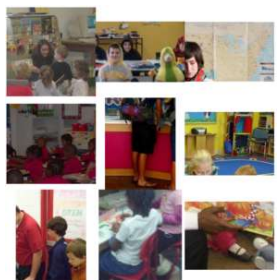

children

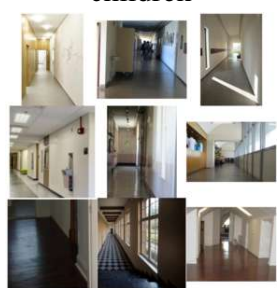

hallway 